\title{
Generation and Characterization of Osterix-Cherry Reporter Mice
}

\author{
Sara Strecker, Yu Fu, Yaling Liu, and Peter Maye* \\ Department of Reconstructive Sciences, School of Dental Medicine, University of Connecticut \\ Health Center
}

\begin{abstract}
Osterix is a zinc finger containing transcription factor, which functions as a key regulator of osteoblast differentiation. To better understand the temporal and spatial expression of Osterix during embryonic development and in the adult skeleton, we generated Osterix-Cherry reporter mice. Bacterial recombination techniques were employed to engineer a transgenic construct, which consisted of a $\sim 39 \mathrm{~kb}$ DNA fragment encompassing the $O$ sterix $/ S p 7$ gene, but excluding adjacent gene sequences. Osterix reporter expression was characterized at embryonic, neonatal, and adult ages both by itself and in the context of a cross with Bone Sialoprotein (BSP)-Topaz reporter mice. Relative to Osterix, BSP is a more mature marker of osteoblast differentiation. In agreement with osteoblast lineage maturation, Osterix reporter expression preceded $B S P$ reporter expression during embryonic development and spatially appeared in a much broader cell population. Strong Osterix reporter expression was observed in mature osteoblasts and osteocytes. However, weaker Osterix-Cherry positive cells were also observed in the bone marrow, possibly identifying an early osteoprogenitor cell population. Evaluation of Osterix reporter expression in male femur tissue sections from 10 days to 12 weeks of age revealed persistent expression in cells of the osteoblast lineage and a surprising increase in maturing chondrocytes of the growth plate. Also, Osterix reporter expression was transiently detected in the kidney after birth.
\end{abstract}

\section{Keywords}

osteoblast; bone; fluorescent; transgenic

\begin{abstract}
Bone development occurs through two different processes termed intramembranous ossification and endochondral ossification (reviewed in: Hall and Miyake, 2000; Mackie et al., 2008; Mackie et al., 2011; Ornitz and Marie, 2002). Most bones of the craniofacial skeleton develop through intramembranous ossification, where mesenchymal cells directly differentiate into cells of the osteoblast lineage. However, in the axial and appendicular skeleton, all bones develop through endochondral ossification, in which mesenchymal cells first condense and differentiate into chondrocytes that form a cartilaginous anlagen. The cartilaginous anlagen serves as a physical template for bone formation and chondrocytes within the anlagen signal to the outer perichondrium, providing critical signaling cues that coordinate the colonization/replacement of cartilage by bone.
\end{abstract}

Genetic studies in mice have revealed that Osterix is a key transcriptional regulator of osteoblast differentiation and, more recently, of chondrocyte maturation. Osterix is a Cys2-

\footnotetext{
"Corresponding Author: Peter Maye, Department of Reconstructive Sciences, MC3705, L7007, School of Dental Medicine, University of Connecticut Health Center, 263 Farmington Avenue, Farmington, CT 06030, Phone: 860-679-7347, Fax: 860-679-2910, pmaye@neuron.uchc.edu.

Website:http://regenerativemedicine.uchc.edu/faculty/bios/maye.html
} 
His2 type zinc finger protein that binds DNA and belongs to the $S P 1$ family of transcription factors (Nakashima et al., 2002). In Osterix global knockout mice, the initial establishment of a cartilaginous skeleton appears grossly normal, but osteoblast differentiation fails to occur, leading to perinatal lethality (Nakashima et al., 2002). Global inactivation of floxed Osterix at postnatal and adult ages using Cag-CreER mice resulted in loss of osteoblasts on the bone surface and progressive deterioration of bone tissue, revealing that Osterix continues to have an essential role in maintaining bone tissue at adult ages (Zhou et al.). Selective deletion of Osterix with Col2a1-Cre has also revealed an important role for Osterix in chondrocyte maturation. Loss of Osterix in chondrocytes resulted in an expanded hypertrophic zone that failed to completely mature, resulting in a significant delay and reduction in vascular invasion and bone remodeling by osteoclasts (Oh et al.).

To increase our understanding of Osterix gene expression at embryonic and adult ages, we generated Osterix-Cherry reporter mice. The Osterix gene is located in a relatively gene rich area on mouse chromosome 15. Therefore, in designing the transgene, we decided to separate Osterix from these neighboring genes by subcloning a $39 \mathrm{~kb}$ DNA fragment containing the Osterix gene using a bacterial recombination approach (Fig. 1A). This subcloned DNA fragment includes $24457 \mathrm{bp}$ of sequence upstream of the first translational start site and ends 6920bp downstream of the stop codon. To verify the isolated region's identity, Not1 and Mlu1 restriction endonuclease digests were carried out. Two predicted bands, one at approximately $22 \mathrm{~kb}$ and the second at $25 \mathrm{~kb}$ were observed, thereby identifying three positive clones (Fig. 1B). Work by others (Milona et al., 2003; Nishio et al., 2006) and our own alignment of mRNA transcripts deposited into the NCBI database have shown that Osterix transcription yields two alternatively spliced mRNAs. $\mathrm{mRNA}_{1}$ encodes for a slightly larger 428 amino acid isoform whose translation starts in exon 1, while $\mathrm{mRNA}_{2}$ encodes for a shorter 410 amino acid isoform whose translation starts in exon 2 (Fig. 1C). The second downstream translation start site is in frame with the upstream translation start site. Therefore, we decided to insert a Cherry fluorescent protein reporter just upstream of the second translation start site in order to detect both Osterix isoforms (Fig. 1C, red arrow head). To confirm the targeted insertion of the Cherry reporter, colony PCR was carried out using primers that flank the 462 bp homology box (Fig. 1D). Pronuclear injection of the DNA construct resulted in generation of two different founder lines. Gross evaluation of reporter gene expression from F1 and F2 offspring from both founder lines showed identical expression patterns (data not shown). Thereafter, we have primarily focused on one founder line and present a more detailed characterization of its reporter gene expression here.

To aid in the characterization of Osterix-Cherry mice, we compared their reporter expression to that of a Bone Sialoprotein (BSP)-Topaz reporter mouse line (Maye et al., 2009). During osteoblast differentiation, Osterix expression precedes $B S P$ and is required for $B S P$ expression (Gordon et al., 2007). The $B S P$-Topaz reporter line utilizes a fluorescent protein that is spectrally distinct from Cherry fluorescent protein, allowing us to simultaneously image both reporters and visualize immature and mature osteoblast cell types. OsterixCherry reporter expression (red) was characterized relative to $B S P$-Topaz reporter expression (green) from days E13.5 to E17.5 of embryonic development. Congruent with skeletal development occurring asynchronously in an anterior to posterior progression, Osterix reporter expression was first detected at E13.5 in craniofacial bones and weakly in the fore limbs (Fig. 1A). BSP reporter expression was also detected at E13.5 (Fig. 2B); however the domains of Osterix reporter expression were noticeably broader than those of the BSP reporter (Fig. 2 Compare A to B, and C). At E15.5, Osterix reporter expression increased relative to E13.5 and now can be detected in the ribs and hind limbs (Fig. 2D). Levels of BSP reporter expression have also increased from E13.5 to E15.5 and globally appear in the same regions as Osterix (Fig. 2E and F). The progression of osteoblast maturation can be appreciated at higher magnification in the frontal and parietal bones of the 
skull (Fig. 2G-I) and the radius, ulna, and humerus of the fore limb (Fig. 2J-K). Again, the domains of Osterix reporter expression are broader than those controlled by BSP and show the transition from osteoprogenitor to a maturing osteoblast. Both Osterix and BSP reporters at this age are osteoblast specific and do not appear in other connective tissue cell types such as the sutures of the skull (Fig. 2G-I) or in joints of the appendicular skeleton (Fig. 2J-L). At E17.5 the expression levels of Osterix and BSP reporters continued to increase (Fig. 2MO). Osterix and $B S P$ reporters are now detected in the vertebrae and pelvic girdle. Also, as limb development progresses in a proximal to distal fashion, Osterix reporter expression is detected in the digits of the paws (Fig. 2M-O).

Characterization of Osterix-Cherry reporter expression was also compared to BSP-Topaz reporter expression in tissue sections from a 3 week old femur (Fig. 3). The whole femur was imaged for both reporters (Fig. 3A) and areas of trabecular bone (Fig. 3B-D) and cortical bone (Fig. 3E-G) are shown at higher magnification. Osterix reporter expression was robustly and broadly expressed in cells of the osteoblast lineage (Fig. 3A). Strong Osterix reporter expression is present in mature osteoblasts lining trabecular and cortical bone surfaces and osteocytes embedded within bone tissue (Fig. 3, A, B, and E). In contrast, $B S P$ reporter expression is not nearly as uniform as the Osterix reporter. High levels of $B S P$ reporter expression were detected just below the growth plate within the trabecular bone region (Fig. 3A and C), but gradually decreased as the distance from the growth plate increased, with lower $B S P$ reporter expression being present in osteoblasts on the cortical bone surfaces in the mid-diaphysis region (Fig. 3 compare $C$ to F). Also, the frequency of $B S P$ positive cells was higher on the endosteal bone surfaces relative to the outer periosteum (Fig. 3F and not shown). In addition to the Osterix reporter being strongly expressed in osteoblasts and osteocytes, we also noted the detection of a bone marrow cell population situated in proximity to bone surfaces that retained lower Osterix reporter expression (Fig. 3B and E). The BSP reporter was not detected in this bone marrow cell population (Fig. 3C and F), suggesting this may be a very early osteoprogenitor cell type.

Loss of function studies at postnatal to adult ages have indicated that Osterix continues to play an essential role in the maintenance of bone tissue (Baek et al.; Baek et al., 2009; Oh et al., 2012; Zhou et al., 2010). Consistent with these studies, the evaluation of Osterix reporter expression in male femurs at increasing ages (10 days, 3 weeks, 8 weeks, and 12 weeks) suggests that Osterix expression persists in cells of the osteoblast lineage into adulthood (Fig. 4). Interestingly, in this temporal analysis we also detected an increase in Osterix reporter expression in maturing chondrocytes of the growth plate. In three week old animals, expression of the Osterix reporter was very weak in the growth plate relative to the level of reporter gene expression detected in the bone cells (Fig 5A). However, by 8 weeks of age, Osterix reporter expression was easily detected in maturing chondrocytes (Fig 5B) and at twelve weeks of age, the area of the growth plate retaining Osterix reporter expression was arguably the highest expressing region within the bone (Fig 5C).

To determine if expression of the Osterix reporter represents endogenous Osterix gene expression, we compared Osterix reporter expression to endogenous levels of Osterix protein by immunostaining. Immunostaining for Osterix was initially carried out directly on bone tissue sections derived from Osterix transgenic mice. However, we soon learned that the polyclonal antibody that worked extremely well in our hands recognized our reporter gene. The reason for this has to do with targeting the Cherry reporter to the second exon just upstream of the second translational start site in order to detect both transcriptional isoforms. In doing so, transcription and translation of $\mathrm{mRNA}_{1}$-Cherry results in the creation of a small protein fusion in which the first 17 amino acids of Osterix are joined to the $\mathrm{N}$-terminal of the Cherry reporter. Correspondence with Santa Cruz Biotechnologies confirmed that the polyclonal antibody (A-13) was raised against this region of Osterix and therefore 
selectively detects the long protein isoform of Osterix. Unfortunately, many other antibodies against Osterix were tried, but did not work well in our hands. Alternatively, we decided to use the A-13 antibody on non-transgenic littermates and compare immunostaining (green fluorescence) to Osterix reporter expression (red fluorescence) (Fig. 6). Tissue sections were counterstained with DAPI (blue fluorescence). Four different regions of interest within the femur were compared; cortical bone (Fig. 6, compare A to B), trabecular bone (Fig. 6, compare $\mathrm{C}$ to $\mathrm{D}$ ), the growth plate (Fig. 6, compare $\mathrm{E}$ to $\mathrm{F}$ ), and the articular surface at the distal end of the femur (Fig. 6, compare $\mathrm{G}$ to H). In the cortical and trabecular bone regions, Osterix reporter expression appeared strongest in osteoblasts lining the bone surface (white arrows), but also had fairly high levels of expression in osteocytes located within the bone matrix (Fig. 6A and C). Consistent with Osterix reporter expression, immunostaining for Osterix was most intense in osteoblasts lining the cortical (white arrows) and trabecular bone surfaces, whereas noticeably less protein was detected in osteocytes (Fig. 6B and D). As detailed earlier, Osterix reporter expression increased with age in maturing chondrocytes within the growth plate (Fig. 5). However, by four weeks of age and relative to osteoblasts and osteocytes, the Osterix reporter is expressed at lower levels in chondrocytes of the growth plate, with slightly higher levels being present in maturing chondrocytes (Fig. 6E). In contrast, immunostained Osterix protein was only detected in chondrocytes undergoing hypertrophy (Fig. 6F). Unlike the differences observed between reporter and immunostaining in the growth plate, Cherry expression correlated well with immunostaining in articular chondrocytes. In the knee joint, Osterix reporter expression is absent from the most superficial zones of the articular surface in the distal femur, but appears in maturing chondrocytes further away from the articular surface (Fig. 6G, white arrows). In a similar manner, endogenous Osterix protein was also detected in maturing chondrocytes below the articular surface (Fig. 6H).

While Osterix immunostaining largely correlated with Osterix reporter gene expression, some differences were observed. Possible reasons for these differences may relate to the fact that the reporter should reflect the expression of both transcriptional isoforms of Osterix, while the A-13 polyclonal antibody only recognized the long protein isoform. Additionally, post-transcriptional mechanisms that would regulate levels of Osterix protein would not be reflected in reporter gene expression.

To further compare expression of the Osterix reporter to endogenous Osterix, we also carried out RT-PCR on reporter positive and negative cell populations that were isolated by FACS from neonatal calvaria and long bones (Fig. 7, A-D). Approximately 50\% of the cells digested out of the calvaria (Fig. 7A) and 12\% of the cells digested from long bones (Fig. 7C) were Osterix reporter positive. RT-PCR was carried out for the reporter (Cherry), the individual isoforms of Osterix ( $\mathrm{mRNA}_{1}$ and $\mathrm{mRNA}_{2}$ ), and GAPDH (Fig. 7E). Amplification for the Cherry reporter confirmed that FACS dramatically enriched for the Osterix reporter positive and negative cell populations. Specific amplification for both alternative isoforms of Osterix revealed that $\mathrm{mRNA}_{1}$ was exclusively expressed in the reporter positive cell population, while $\mathrm{mRNA}_{2}$ could be detected in both cell fractions with higher levels being present in the reporter positive cell fraction.

While expression of the Osterix reporter was largely restricted to skeletal tissues, we also detected high levels of reporter expression in the kidneys of newborn pups, but the signal gradually decreased to a very low level by the time animals were weaned (Fig. 8). To further characterize renal Osterix reporter expression, kidney samples were collected from transgenic mice at E17.5 (Fig. 8A), P4 (Fig. 8B) and 3 weeks (Fig. 8C) of age and the intensity of fluorescent reporter expression was compared. Surprisingly, at E17.5 and 3 weeks, reporter expression was detectable, but very weak, while reporter expression at P4 was extremely high. We also examined Osterix reporter expression in 6 week and 11 week 
old transgenic animals, but no reporter expression was detected (data not shown). Further examination of Osterix reporter expression in tissue sections of P4 animals (Fig. 8, D, E, E', $\mathrm{E}^{\prime \prime}$ ) revealed that the signal was present in the juxtamedullary nephrons, which are located in the cortex, but adjacent to the medulla (Fig. 8D). Within the juxtamedullary nephrons, Osterix reporter expression was detected at low levels in the proximal convoluted tubules and at higher levels in the thick descending limb of the loop of Henle (Fig. 8, $\mathrm{E}^{\prime}$ and $\mathrm{E}^{\prime \prime}$ ).

To determine if Osterix is expressed transiently in the kidney, RT-PCR was carried out on transgenic positive and negative kidney samples at E17.5, P4, and 3 weeks of age (Fig. 8F). Interestingly, we were able to detect only one of the two isoforms in the kidney, $\mathrm{mRNA}_{2}$. Also, expression of the $\mathrm{mRNA}_{2}$ isoform was transient, similar to that observed with our reporter gene. For reasons that remain unclear to us, we had a very difficult time detecting mRNA for the Cherry reporter in the kidney at P4, near the time point where we are able to visualize robust fluorescent expression.

In this study, we have described the transgene assembly and expression of an Osterix-Cherry fluorescent reporter mouse model. Characterization of reporter expression suggests that the $39 \mathrm{~kb}$ transgene largely retains many of the cis regulatory elements necessary for regulating Osterix gene expression in skeletal tissues from embryonic to adult ages. Consistent with Osterix having an essential role in osteoblast and chondrocyte development, Osterix reporter expression was largely restricted to both of these skeletal lineages. However, we have also noted transient reporter expression in the kidney that was particularly strong at neonatal ages. Given the timing of reporter expression in the kidney in relation to lactation, it is intriguing to speculate that perhaps Osterix may have a role in regulating mineral homeostasis during this period of active skeletal growth and development.

The transgenic reporter was designed to detect both transcriptional isoforms of Osterix. Other groups have reported on the existence and expression of both Osterix isoforms, however, there is disagreement with regard to which isoform may be the predominant form, if any. Milona et al. have reported that the shorter isoform $\left(\mathrm{mRNA}_{2}\right)$ is more highly expressed in skeletal cell types (Milona et al., 2003), while Nishio et al. provided evidence that the longer isoform $\left(\mathrm{mRNA}_{1}\right.$ ) predominates (Nishio et al., 2006). Our immunostaining studies, which utilized an antibody that recognizes the long protein isoform of Osterix clearly indicate that $\mathrm{mRNA}_{1}$ is highly expressed in osteoblasts and hypertrophic chondrocytes. However, analyses of isoform specific transcripts by RT-PCR from FACS isolated cell populations indicate that both transcriptional isoforms are likely to be expressed in a relatively skeletal specific manner with perhaps $\mathrm{mRNA}_{2}$ being expressed at significantly lower levels in non-skeletal cell types. It remains unclear if any functional differences exist between either protein isoform of Osterix.

One of the significant advantages of fluorescent protein reporter mice is that they provide investigators with the means to isolate and study intrinsically labeled cell populations. Work by us and others have generated a variety of different fluorescent reporter mice to study the osteoblast lineage (Kalajzic et al., 2004; Kalajzic et al., 2002a; Kalajzic et al., 2002b; Maye et al., 2009). As a critical mediator of osteoblast commitment, Osterix has a very early and pivotal role within the osteoblast lineage. Supporting this thinking, the onset of Osterixdriven Cherry expression appeared earlier and in a broader cell population than BSP reporter expression during embryonic development. We also observed a low Osterix reporter expressing cell population in the bone marrow that was also BSP reporter negative, which may mark a very early osteoprogenitor required for supporting postnatal skeletal growth and bone maintenance. Therefore, Osterix-Cherry reporter mice may provide us with a means to isolate and study the earliest cells of the osteoblast cell lineage, which still remain poorly understood. 


\section{Materials and Methods \\ DNA Constructs and Animal Care}

BAC clone RP24-362M3 was obtained from the Children's Hospital Research Institute (CHORI). The BACLinkSP linking vector was generously provided by Claire Huxley (Kotzamanis and Huxley, 2004). The pLD53-SC2 and pSV1.RecA recombination vectors were generously provided by Shiaoching Gong (Gong et al.). The mini lambda vector was generously provided by Donald M. Court (Court et al., 2003). Transgenic animals were housed in a clean barrier facility and humanely treated in accordance with University of Connecticut Health Center institutional guidelines. The Osterix-Cherry transgenic mouse line will become available to the research community upon acceptance of this manuscript.

\section{Cloning Homology Arms into the BAC Linking Vector}

All homology arms were amplified using Phusion DNA Polymerase (New England BioLabs) in a C1000 Thermal Cycler (BioRad). An overlapping PCR scheme was used to simultaneously clone homology arms flanking the Osterix gene into BACLink SP. For this, homology arm 1 (A1) was amplified using oligos 5'Arm1OLPCR (sense) $5^{\prime}$ -

AGCTAAGGCTGGGCTTTCTTGATTTGTCTGGTGTGCATGCACCACCATGCCCAGT G ACAA- $3^{\prime}$ and $3^{\prime}$ Arm1Mlu (antisense) $5^{\prime}$ CTTCACGCGTCACTAACAAACGCTTCTATAATCCTTAC- $3^{\prime}$ which contained an Mlu1 site. Homology arm 2 (A2) was amplified using 5' Arm2BamH1 (sense) $5^{\prime}$ -

CTCTGGATCCGAGGGAGGAGGATCTTGACCAGCATCA-3' which contained a BamH1 site and 3' OLPCRArm2 (antisense) 5' TTGTCACTGGGCATGGTGGTGCATGCACACCAGACAAATCAAGAAAGCCCAGCC T AGCT-3'. PCR amplified homology arms were run out on a 1\% agarose gel and gel purified using a Zymoclean Gel DNA Recovery Kit (Zymo Research). The purified PCR products were eluted in 10ul of water and diluted 1:10 in water and combined into a single tube. To promote annealing of the overlapping region, homology arms were heated to $95^{\circ} \mathrm{C}$ and a seven minute cool down was implemented followed by thermal cycling $\left(95^{\circ} \mathrm{C}-55^{\circ} \mathrm{C}-72^{\circ} \mathrm{C}\right)$ for 25 cycles. After 25 cycles, $5^{\prime}$ Arm2BamH1 or $3^{\prime}$ Arm1Mlu1 primers were added to the reaction and it was allowed to proceed for an additional 20 cycles. The resultant band was gel purified, restriction endonuclease digested with BamH1 and Mlu1, and cloned into the BamH1 and Mlu1 sites of pBACLinkSP using conventional cloning practices to create BACLinkSP-A1/A2.

\section{Subcloning into BACLinkSP-A1/A2 to Create BACLinkSP-Osterix}

The Red Recombinase System was introduced into RP24-362M3 containing DH10B cells by electroporation of a mini lamda vector (Court et al., 2003; Gong et al., 2002; Liu et al., 2003; Wang et al., 2006). Transformants were selected on chloramphenicol (12.5 ug/ml) plus tetracycline (10ug/ml) LB agar plates and grown at $30^{\circ} \mathrm{C}$. RP24-362M3 and minilambda-containing DH10B cells were made electrocompetent. Prior to harvesting cells to make them electrocompetent, bacteria were grown at $30^{\circ} \mathrm{C}$ and heat shocked at $42^{\circ} \mathrm{C}$ for 15 minutes to activate the Red Recombinase System.

To subclone the Osterix gene into BACLinkSP-A1/A2, Xho1 was used to digest BACLinkSP-A1/A2 and expose the ends of homology arms 1 and 2 . The linearized vector was then electroporated into RP24-362M3 and mini-lambda containing cells and selected for on LB agar plates containing spectinomycin $(50 \mathrm{ug} / \mathrm{ml})$. Recombinants were screened by colony PCR with five separate primer pairs. Two primer pairs were designed to flank homology arms A1 and A2. Primer pairs flanking homology arm 1 were : Ost $5^{\prime}$ Arm1 Recom (sense) 5' ${ }^{\prime}$-CTACATGATGATTCAGAACCATCTGTAACT-3' and Belo2658 (antisense) $5^{\prime}$-TTTGTCACAGGGTTAAGGGC-3'. Primer pairs flanking 
homology arm 2 were Ost 3'Arm2Recom (antisense) $5^{\prime}$ -

CAGACAAATCAAGAAAGCCCAGCCTTAGCT- $3^{\prime}$ and SP2 sense $5^{\prime}$ -

GCCCTACACAAATTGGGAGA- $3^{\prime}$. The three remaining primer pairs amplified different regions within the Osterix subclone region and were used to confirm that the entire region was transferred. These three primer pairs were OstSub5 $5^{\prime} \mathrm{T} 1$ (sense) $5^{\prime}$ -

TGGTCCAAGCCTGTGGACCAAGAAGCA-3' and OstSub3' T1 (antisense) 3' AGGAACCACCTACTGAGAGGTGGCTAT- $5^{\prime}$; OstSub5'T2ig (sense) $5^{\prime}$ TCATAATGTTTCCGTGTCACCATC-3' and OstSub3'T2ig (antisense) $3^{\prime}$ TGCATGCGCTCTTGTGCATATGTACAT-5'; OstSub5'T3 (sense) 5' CTTAGCAGACACATACCCGAGGATGA-3' and OstSub3'T3 (antisense) $3^{\prime}$ ACTGAGTCCTCTGCACCAGTTGTAAG-5' . BAC subclones positively identified by colony PCR were then further verified by Not1 and Mlu1 restriction endonuclease digestion followed by field inversion gel electrophoresis.

\section{Homology Arm Cloning into pLD53 to Create pLD53-Cherry-Osterix}

A 462bp homology arm located just upstream of the second translational start site of Osterix was inserted into a pLD53.SC2-Cherry vector using standard cloning practices. The homology arm was PCR amplified using Phusion DNA polymerase using the RP24-362M3 $\mathrm{BAC}$ as a template. Primers Ost5 $5^{\prime}$ Asc 1 (sense) $5^{\prime}$ CTCTGGCGCGCCGTAGCTGAGGATGACCTGAGGTTC-3' and Ost3'Sma1 (antisense) $3^{\prime}$-CTCTCCCGGGGACTGGAGCCATAGTGAGCTTCTTC-5' were used to amplify the homology arm. Amplified products were run through a PCR clean up column (Qiagen) to reduce unincorporated nucleotides, then restriction endonuclease digested with Asc1 and Sma1 for three hours. The pLD52.SC2-Cherry vector was also restriction endonuclease digested with Acs1 and Sma1 for three hours. After digestion, the homology arm was gel purified using a Zymoclean Gel DNA Recovery Kit (Zymo Research). The pLD53.SC2Cherry vector was briefly treated with calf intestinal alkaline phosphatase (New England BioLabs) followed by a 1:1 phenol:chloroform extraction and precipitation. Vector and insert were mixed and ligated at room temperature for an hour using Quick Ligase (New England Biolabs) followed by electroporation into competent PIR2 cells. Bacteria were allowed to recover for a half hour before selection. Possible clones were selected for on LB plates containing ampicillin $(50 \mathrm{ug} / \mathrm{ml})$ and identified by colony PCR screening followed by diagnostic restriction endonuclease digestion with Asc1 and Sma1.

\section{Reporter Gene Insertion to Create BACLinkSP-Osterix-Cherry}

In preparation for reporter gene insertion into BACLinkSP-Osterix, 50ng of pSV1.RecA was transformed into DH10B cells containing BACLinkSP-Osterix. After transformation, bacteria were allowed to recover for one hour without antibiotic selection followed by selection on LB agar plates containing spectinomycin $(50 \mathrm{ug} / \mathrm{ml})$ and tetracycline $(10 \mathrm{ug} / \mathrm{ml})$ at $30^{\circ} \mathrm{C}$ overnight. DH10B cells containing BACLinkSP-Osterix and pSV1.RecA were made electrocompetent.

pLD53.Sc2-Cherry-Osterix (1ug) was electroporated into 50ul of electrocompetent bacteria containing the pSV1 vector and BACLinkSP-Osterix. Bacteria were grown in $1 \mathrm{ml}$ of LB without antibiotic selection for 1 hour at $30^{\circ} \mathrm{C} .5 \mathrm{ml}$ of $\mathrm{LB}$ media containing spectinomycin $(50 \mathrm{ug} / \mathrm{ml})$, ampicillin $(50 \mathrm{ug} / \mathrm{ml})$ and tetracycline $(10 \mathrm{ug} / \mathrm{ml})$ were then added to the electroporated cells and the culture was grown overnight at $30^{\circ} \mathrm{C} .200 \mathrm{ul}$ of the overnight culture was spread on to an agar plate containing spectinomycin $(50 \mathrm{ug} / \mathrm{ml})$ and ampicillin (50ug/ml) and grown at $42^{\circ} \mathrm{C}$ overnight. Colonies were picked and screened using primers that flanked the homology arm; OsterixRecom (sense) $5^{\prime}$ GCCATCACACCAAGCCTGCTTTGTGT-3' and CherryGenotype (antisense) $5^{\prime}$ GCACCTTGAAGCGCATGAACTCCTTGATGA-3'. Potential BACLinkSP-Osterix- 
Cherry clones which were positively identified by PCR were then further verified by diagnostic restriction endonuclease digestion followed by field inversion gel electrophoresis.

\section{Preparation of BACLinkSP-Osterix-Cherry for Pronuclear Injection}

Verified BACLinkSP-Osterix-Cherry clones were grown and purified from $200 \mathrm{ml}$ of bacterial culture using a Maxi kit (Qiagen) with minor modifications detailed here. After alkaline lysis $2 \mathrm{M}$ potassium acetate was used in place of the standard $3 \mathrm{M}$ solution. $\mathrm{QF}$ buffer was heated to $65^{\circ} \mathrm{C}$ for elution and the column eluate was further purified with a 1:1 phenol:chloroform extraction followed by a chloroform extraction. 10ug of the purified BACLinkSP-Osterix-Cherry was linearized with a Sal1 restriction enzyme digest and further purified on a Sepharose CL-4B column (Sigma) that had been equilibrated with injection buffer (10mM Tris pH 7.5, 0.1mM EDTA, $100 \mathrm{mM} \mathrm{NaCl})$. Ten 200ul fractions were collected and the DNA was quantified using a NanoDrop spectrophotometer (Thermo Scientific). $30 \mathrm{ul}$ of each fraction was run on a pulse field gel to assess DNA quality. Pronuclear injection was carried out at the UCONN Health Center Gene Targeting and Transgenic Facility (GTTF).

\section{Histological Preparation of Tissue Samples}

Selected tissues were dissected and fixed in 10\% Formalin buffered in PBS for 4 days at $4^{\circ} \mathrm{C}$. Hind limbs from two week old or older mice were decalcified in 15\% EDTA for 4 to 7 days, depending on animal age. Tissues were then placed in 30\% sucrose overnight and finally embedded in Cryomedia (Thermo Scientific). Frozen $7 \mathrm{um}$ sections were obtained using a Leica Cryostat and Cryofilm type II tape transfer system (Section-Lab Co. Ltd.). Sequential tissue sections were mounted using 50\% glycerol buffered in PBS for imaging.

\section{Microscopy and Imaging}

Whole mount images of embryonic animals and kidneys were taken using a Zeiss SteREO Lumar V.12 fluorescent microscope at 9.6X and 15.4X magnification using Cherry (HQ577/20 Ex, HQ640/40 Em) and EYFP (ET500/20 Ex, ET535/30 Em) filter sets (Chroma Technologies) and photographed with an Axiocam MRm digital camera (Zeiss). Exposure times were adjusted for optimum imaging, and kept consistent throughout the experimental time course. Tissue sections were imaged on a Zeiss Observer Z.1 microscope using Cherry (HQ577/20 Ex, HQ640/40 Em, Q595lp beam splitter) and EYFP (HQ500/20 Ex; HQ 535/30 Em, Q515lp beam splitter) filter sets (Chroma Technologies). The same tissue sections were then stained with Mayer's hematoxylin (Poly Scientific) and photographed using an Axiocam MRc digital camera (Zeiss) also on the Zeiss Observer Z.1 microscope.

\section{Immunostaining for Osterix}

Tissue sections were dried for 30 minutes at room temperature and then rehydrated by rinsing in PBS for 15 minutes. Sections were then permeabilized for 30 minutes in $0.1 \%$ Triton X-100 (Sigma) at room temperature. Afterwards, sections were washed twice for 10 minutes in PBS at room temperature. Non-specific staining was blocked with a $1 \%$ BSA and 5\% goat serum solution (Invitrogen) in PBS for one hour. The blocking solution was removed, and sections was incubated in an Osterix rabbit polyclonal $\mathrm{IgG}$ primary antibody (Santa Cruz Biotechnologies A-13, sc-22536) at a 1:2000 dilution in 1\% BSA and 1\% goat serum in PBS overnight at $4^{\circ} \mathrm{C}$. Tissue sections were then washed three times (10 minutes per wash in PBS) and suspended in a solution containing the secondary antibody, goat antirabbit Alexa Fluor 488 (Invitrogen) at a 1:500 dilution in 1\% BSA and 1\% goat serum in PBS. Tissue sections were incubated with the secondary antibody at room temperature in the dark for two hours. Sections were then washed three times for ten minutes each in PBS and mounted on slides in a 50\% glycerol/ PBS solution for imaging. 


\section{Harvesting and FACS Isolation of Primary Cells from Calvaria and Long Bone}

Five to seven day old mice were sacrificed and dissected to obtain their skulls and hind limbs. Soft tissues were grossly removed with a scalpel and calvaria and hind limb long bones were placed in ice cold PBS. Cells were enzymatically digested from bone tissue in a solution containing $0.625 \mathrm{mg} / \mathrm{ml}$ Collagenase P (Roche) and $0.01 \%$ Trypsin EDTA (Invitrogen) in PBS. Bone tissues were initially digested for 15 minutes in a 37oC shaker, and the supernatant from the first digestion was discarded. Three subsequent digestions were carried out for 20 minutes each. Cells from these three digestions were pooled, run through a $70 \mu \mathrm{M}$ strainer, centrifuged at 300xg and resuspended in 49\% PBS, 49\% OPTI-MEM (Gibco) and 2\% FBS. Prior to FACS sorting, cells were filtered again through a $5 \mathrm{ml}$ round bottom $40 \mu \mathrm{M}$ cell strainer capped FACS tube (Falcon) and kept on ice. FACS isolation was carried out using a Vantage SE FACS sorter (BD Biosciences) at the University of Connecticut Health Center Flow Cytometry Facility. Cells were collected in 20\% serum in a 1:1 mixture of OPTI-MEM (Gibco) and PBS on ice.

\section{RNA extraction}

A Nucleospin RNA/Protein Kit (Macherey-Nagel) was used to extract the RNA from sorted cell populations. In brief, cells were lysed in RA1 buffer containing B-mercaptoethanol. The resultant mixture was filtered through a column, in which the cell lysate was cleared. Ethanol was added to the filtrate and the mixture was spun through a second column, where the RNA binds to the column matrix and proteins flow through. The column membrane was desalted and then the DNA was digested, yielding a pure RNA sample in the column. The column was then washed twice with ethanol and eluted in 40ul of RNase-free water. RNA concentrations were measured with a NanoDrop spectrophotometer (Thermo Scientific).

RNA was extracted from kidneys at different ages. Isolated kidneys were placed in Trizol (Invitrogen) and homogenized. Following the chloroform extraction, RNA was further purified through consecutive 1:1 phenol:chloroform extractions until the interface between aqueous and organic layers appeared clean. Samples were then chloroform extracted and precipitated in isopropanol. RNA pellets were washed in $75 \%$ ethanol and resuspended in DEPC treated water. RNA concentrations were measured with a NanoDrop spectrophotometer (Thermo Scientific) and RNA integrity was confirmed by gel electrophoresis.

\section{cDNA Synthesis}

RNA samples were first treated with DNAse I (Invitrogen) for 15 minutes at room temperature to remove possible genomic DNA contamination. 25mM EDTA (Invitrogen) was added to each sample and samples were incubated at $65^{\circ} \mathrm{C}$ for 10 minutes to inactive DNAse I. A master mix containing first strand buffer, random primers, DTT, and dNTPs were added to each sample. Samples were then incubated for 10 minutes at $65^{\circ} \mathrm{C}$ followed by incubation on ice. RNaseOUT recombinant ribonuclease inhibitor (Invitrogen) and MMLV reverse transcriptase (Invitrogen) were added to each tube. Samples were mixed and incubated at $37^{\circ} \mathrm{C}$ for 1.5 hours, then the M-MLV was heat inactivated at $85^{\circ} \mathrm{C}$ for 10 minutes.

\section{PCR Amplification for Osterix mRNA Isoforms}

mRNA isoform specific primers were designed using Vector NTI software (Invitrogen) and the NCBI database / Primer-BLAST. mRNA $_{1}$ was selectively amplified using primer sets: Exon1-2 5' (sense) 5' -TTCTCTCCATCTGCCTGACTCCTT-3'', Exon1-2 3' (antisense) $5^{\prime}$-CCATTGGTGCTTGAGAAGGGAGCTG-3' ${ }^{\prime}$. mRNA $_{2}$ was selectively amplified using primer sets: Exon2 5' (sense) 5' -AGGCCACCCATTGCCAGTAA-3', Exon $23^{\prime}$ 
(antisense) $5^{\prime}$-GTCATTTGCATAGCCAGAGGCTGG-3' ${ }^{\prime}$. PCR amplification was also carried out for the Cherry reporter gene: Cherry $25^{\prime}$ (sense) $5^{\prime}$ -

CATCCCCGACTACTTGAAGC- $3^{\prime}$ and Cherry2 $3^{\prime}$ (antisense) $5^{\prime}$ -

CTTCAGCTTCAGCCTCTGCT-3' and GAPDH: GAPDH5' (sense) 5-

CATGTTCCAGTATGACTCCACTC-3' and GAPDH3' (antisense) $5^{\prime}$ -

GGCCTCACCCCATTTGATGT-3' ${ }^{\prime}$. PCR amplification was carried out using standard

methodologies using a C1000 Thermal Cycler (BioRad).

\section{Acknowledgments}

The authors would like to thank Dr. David Rowe for sharing the necessary equipment required to characterize Osterix reporter mice, Dr. Mark Kronenberg for critically reviewing this manuscript, and Dr. Thiruchandurai V. Rajan for clarifying the anatomical localization of reporter expression within the kidney. This work was supported by a grant from NIAMS (1R21 AR060899) and the NIDCR (T32 DE007302-15 and T90 DE021989-01)

\section{References}

Baek WY, de Crombrugghe B, Kim JE. Postnatally induced inactivation of Osterix in osteoblasts results in the reduction of bone formation and maintenance. Bone. 46:920-928. [PubMed: 20026264]

Baek WY, Lee MA, Jung JW, Kim SY, Akiyama H, de Crombrugghe B, Kim JE. Positive regulation of adult bone formation by osteoblast-specific transcription factor osterix. J Bone Miner Res. 2009; 24:1055-1065. [PubMed: 19113927]

Court DL, Swaminathan S, Yu D, Wilson H, Baker T, Bubunenko M, Sawitzke J, Sharan SK. Minilambda: a tractable system for chromosome and BAC engineering. Gene. 2003; 315:63-69. [PubMed: 14557065]

Gong S, Kus L, Heintz N. Rapid bacterial artificial chromosome modification for large-scale mouse transgenesis. Nat Protoc. 5:1678-1696. [PubMed: 20885380]

Gong S, Yang XW, Li C, Heintz N. Highly efficient modification of bacterial artificial chromosomes (BACs) using novel shuttle vectors containing the R6Kgamma origin of replication. Genome Res. 2002; 12:1992-1998. [PubMed: 12466304]

Gordon JA, Tye CE, Sampaio AV, Underhill TM, Hunter GK, Goldberg HA. Bone sialoprotein expression enhances osteoblast differentiation and matrix mineralization in vitro. Bone. 2007; 41:462-473. [PubMed: 17572166]

Hall BK, Miyake T. All for one and one for all: condensations and the initiation of skeletal development. Bioessays. 2000; 22:138-147. [PubMed: 10655033]

Kalajzic I, Braut A, Guo D, Jiang X, Kronenberg MS, Mina M, Harris MA, Harris SE, Rowe DW. Dentin matrix protein 1 expression during osteoblastic differentiation, generation of an osteocyte GFP-transgene. Bone. 2004; 35:74-82. [PubMed: 15207743]

Kalajzic I, Kalajzic Z, Kaliterna M, Gronowicz G, Clark SH, Lichtler AC, Rowe D. Use of type I collagen green fluorescent protein transgenes to identify subpopulations of cells at different stages of the osteoblast lineage. J Bone Miner Res. 2002a; 17:15-25. [PubMed: 11771662]

Kalajzic Z, Liu P, Kalajzic I, Du Z, Braut A, Mina M, Canalis E, Rowe DW. Directing the expression of a green fluorescent protein transgene in differentiated osteoblasts: comparison between rat type I collagen and rat osteocalcin promoters. Bone. 2002b; 31:654-660. [PubMed: 12531558]

Kotzamanis G, Huxley C. Recombining overlapping BACs into a single larger BAC. BMC Biotechnol. 2004; 4:1. [PubMed: 14709179]

Liu P, Jenkins NA, Copeland NG. A highly efficient recombineering-based method for generating conditional knockout mutations. Genome Res. 2003; 13:476-484. [PubMed: 12618378]

Mackie EJ, Ahmed YA, Tatarczuch L, Chen KS, Mirams M. Endochondral ossification: how cartilage is converted into bone in the developing skeleton. Int J Biochem Cell Biol. 2008; 40:46-62. [PubMed: 17659995]

Mackie EJ, Tatarczuch L, Mirams M. The skeleton: a multi-functional complex organ: the growth plate chondrocyte and endochondral ossification. J Endocrinol. 2011; 211:109-121. [PubMed: 21642379] 
Maye P, Stover ML, Liu Y, Rowe DW, Gong S, Lichtler AC. A BAC-bacterial recombination method to generate physically linked multiple gene reporter DNA constructs. BMC Biotechnol. 2009; 9:20. [PubMed: 19284652]

Milona MA, Gough JE, Edgar AJ. Expression of alternatively spliced isoforms of human Sp7 in osteoblast-like cells. BMC Genomics. 2003; 4:43. [PubMed: 14604442]

Nakashima K, Zhou X, Kunkel G, Zhang Z, Deng JM, Behringer RR, de Crombrugghe B. The novel zinc finger-containing transcription factor osterix is required for osteoblast differentiation and bone formation. Cell. 2002; 108:17-29. [PubMed: 11792318]

Nishio Y, Dong Y, Paris M, O'Keefe RJ, Schwarz EM, Drissi H. Runx2-mediated regulation of the zinc finger Osterix/Sp7 gene. Gene. 2006; 372:62-70. [PubMed: 16574347]

Oh JH, Park SY, de Crombrugghe B, Kim JE. Chondrocyte-specific ablation of Osterix leads to impaired endochondral ossification. Biochem Biophys Res Commun. 418:634-640. [PubMed: 22290230]

Ornitz DM, Marie PJ. FGF signaling pathways in endochondral and intramembranous bone development and human genetic disease. Genes Dev. 2002; 16:1446-1465. [PubMed: 12080084]

Wang J, Sarov M, Rientjes J, Fu J, Hollak H, Kranz H, Xie W, Stewart AF, Zhang Y. An improved recombineering approach by adding RecA to lambda Red recombination. Mol Biotechnol. 2006; 32:43-53. [PubMed: 16382181]

Zhou X, Zhang Z, Feng JQ, Dusevich VM, Sinha K, Zhang H, Darnay BG, de Crombrugghe B. Multiple functions of Osterix are required for bone growth and homeostasis in postnatal mice. Proc Natl Acad Sci U S A. 2010; 107:12919-12924. [PubMed: 20615976] 
A

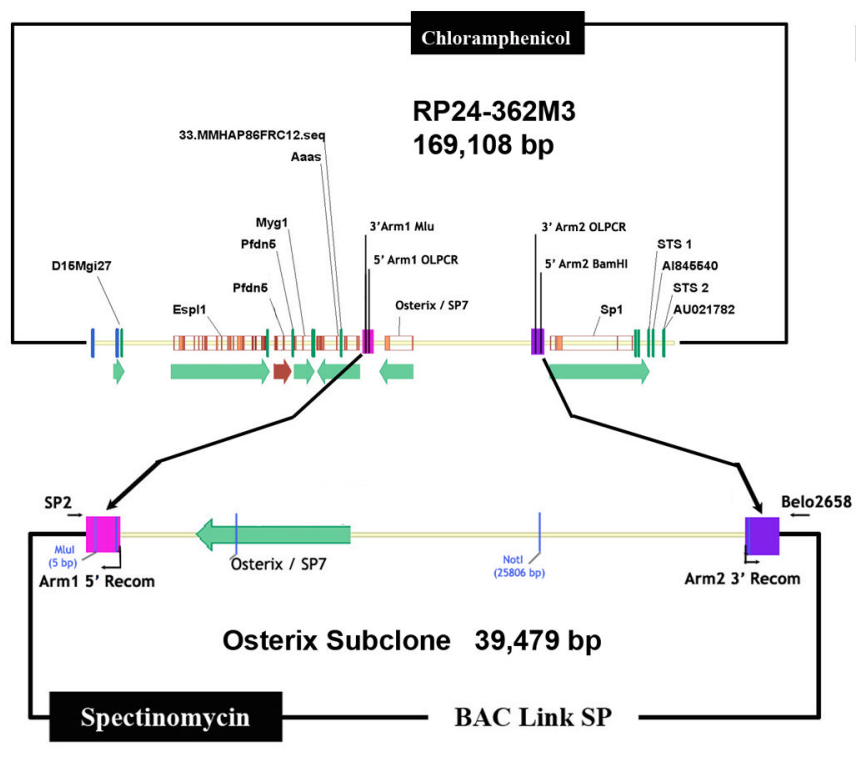

C

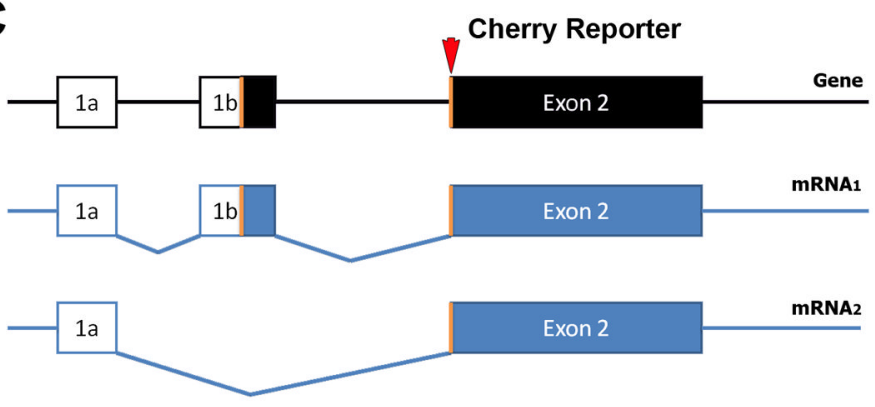

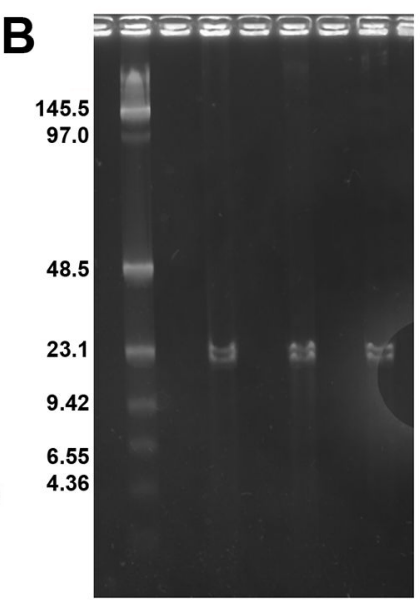

D

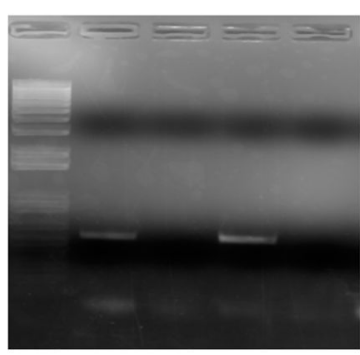

FIG. 1. Assembly of the Osterix-Cherry Transgene

(a) A $39 \mathrm{~kb}$ region containing the Osterix gene and flanking DNA sequences was subcloned from BAC clone RP24-362M3 into BACLink-SP. (b) Potential subclones were confirmed by a diagnostic restriction endonuclease digestion with Mlu1 and Not1, which showed two DNA fragments at $25 \mathrm{~kb}$ and $22 \mathrm{~kb}$. (c) Diagram of the Osterix gene (black) mapped with two alternatively spliced mRNA transcripts (blue). Two alternative translational start sites also exist. The first translational start site is located in exon $1 \mathrm{~b}$ and results in the creation of a long protein isoform $\left(\mathrm{mRNA}_{1}\right)$ consisting of 428 amino acids. The second translational start site is located near the beginning of exon 2 , is in frame with the upstream translational start site, and results in the creation of a shorter protein isoform $\left(\mathrm{mRNA}_{2}\right)$ consisting of 410 amino acids. The Cherry reporter was inserted via homologous recombination just upstream of the second translational start site of the Osterix gene (red arrow) allowing us to detect both transcriptional isoforms. (d) Gel image of colony PCR screening to identify clones where the Cherry reporter was appropriately inserted into the construct. Two positive clones are shown. 


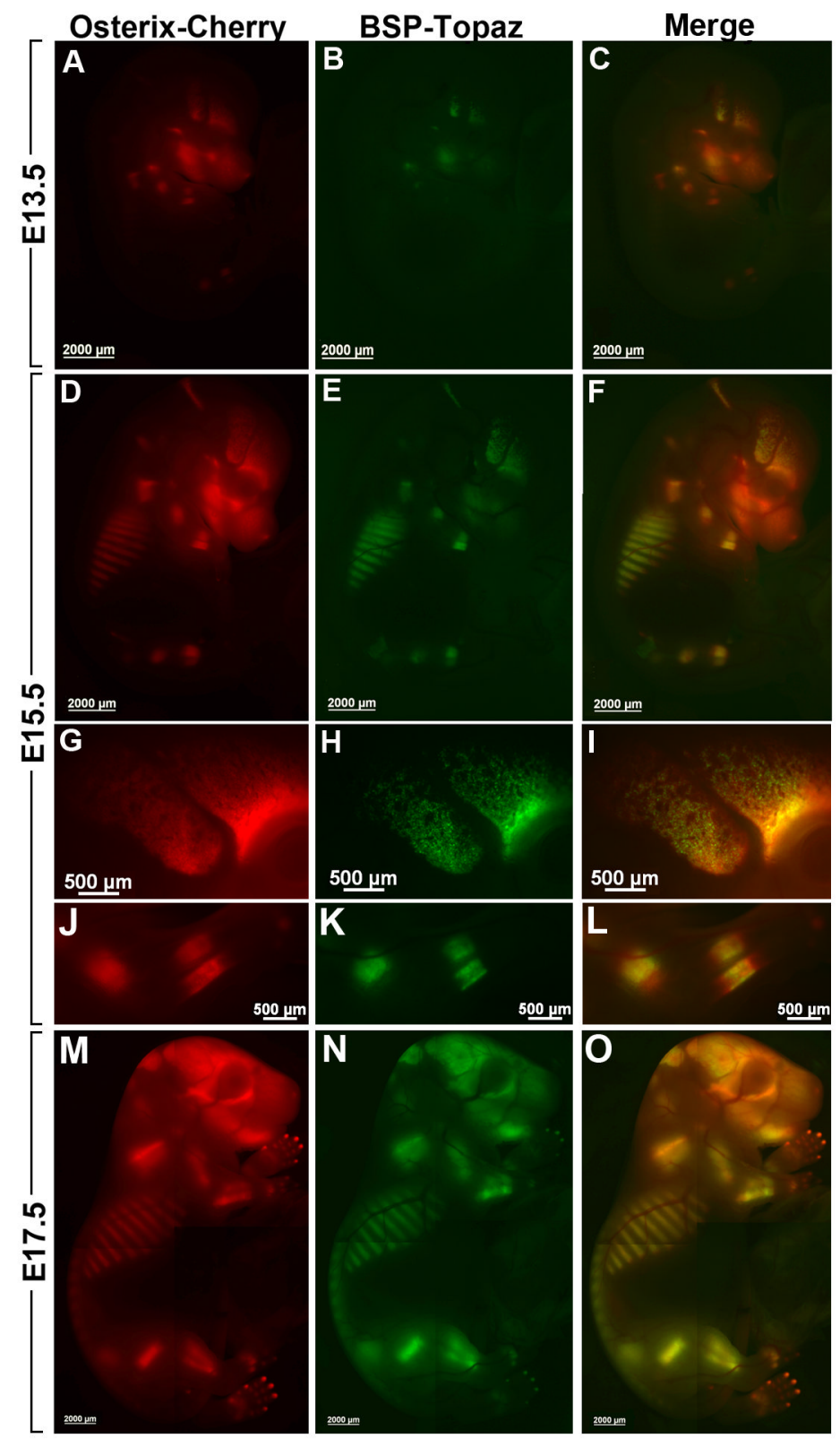

FIG. 2. Comparison of Osterix reporter expression to Bone Sialoprotein reporter expression during embryonic skeletal development

(a-o) Whole embryo imaging of reporter gene expression from E13.5 -E17. (a,d,g,j,m) Osterix-Cherry reporter expression (red). (b,e,h,k,n) Bone Sialoprotein - Topaz reporter expression (green). (c,f,i,l, ,o) Merge of Osterix and Bone Sialoprotein reporter expression (yellow indicates where the signals overlap). (a-c) Comparison of Osterix and Bone Sialoprotein reporter expression at E13.5 (a) Osterix reporter expression is first detected at E13.5 in the developing craniofacial bones and in the fore limbs. Very weak Osterix reporter expression is detected in the developing hind limbs. (b) The onset of Bone Sialoprotein reporter expression is also detected at E13.5. (c) The merged image confirms the overlap between both osteoblast reporter models, however the Osterix reporter is expressed in a considerably broader cell population than the Bone Sialoprotein reporter. (d-i) Comparison of Osterix and Bone Sialoprotein reporter expression at E15.5. (d) Levels of Osterix reporter 
expression have increased relative to E13.5 and are now easily detected in the hind limbs and ribs in addition to the bones of the craniofacial skeleton and fore limb. (e) Levels of Bone Sialoprotein reporter expression have also increased since E13.5 and appear in the same skeletal elements as the Osterix reporter as shown in the merged image (f). While both reporter genes are expressed in the same skeletal elements, the broader cellular expression of the Osterix reporter relative to the Bone Sialoprotein reporter is apparent and appreciated at higher magnification in the skull $(\mathbf{g}-\mathbf{i})$ and fore limb $(\mathbf{j}-\mathbf{l})$. (m-0) Osterix and Bone Sialoprotein reporter expression at E17.5. Osterix reporter expression is now detected in the vertebrae and developing digits, whereas the Bone Sialoprotein reporter is seen in the spine, but has not yet appeared in the metatarsals. 

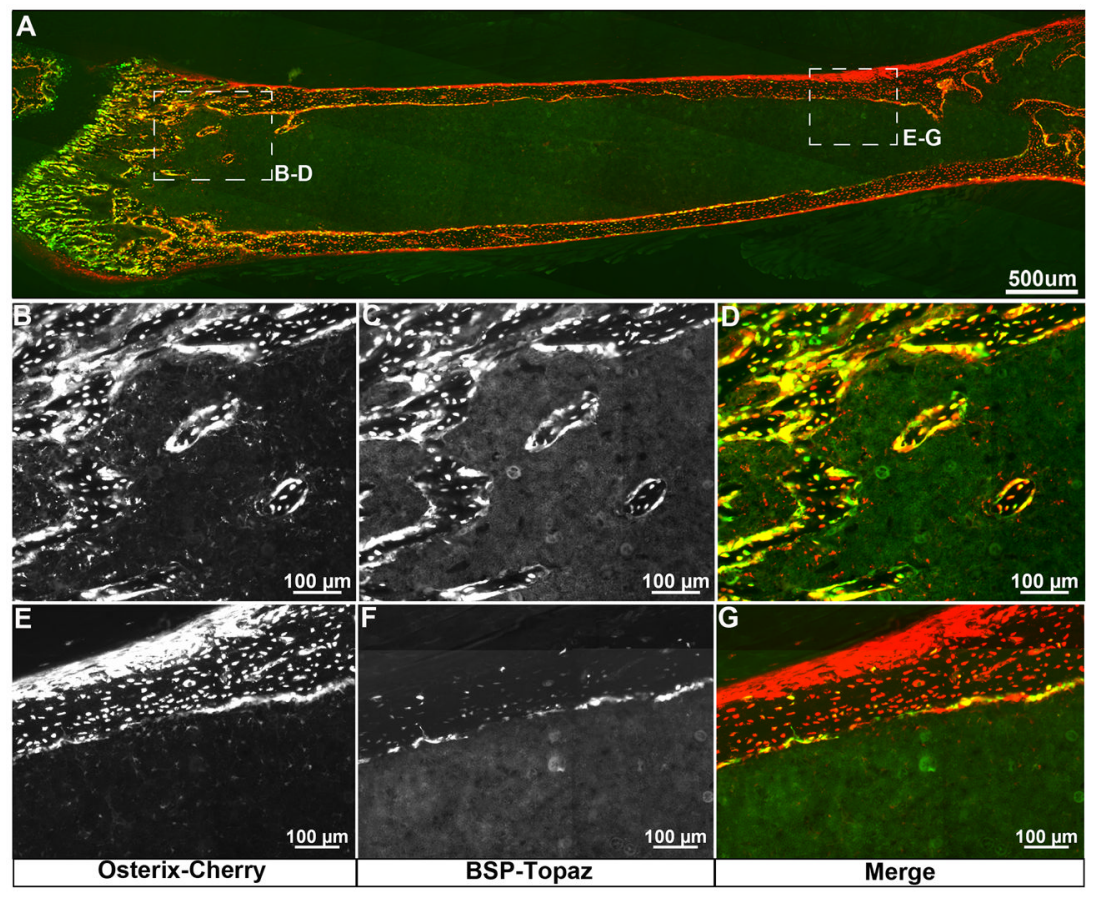

FIG. 3. Comparison of Osterix reporter expression to Bone Sialoprotein reporter expression in a tissue section through a 3 week old femur

(a) Merged image of Osterix-Cherry (red) and Bone Sialoprotein-Topaz (green) reporter expression. Global visualization of reporter expression revealed that the Osterix reporter is intensely and uniformly expressed in osteoblasts within the trabecular and cortical bone regions. In contrast, Bone Sialoprotein reporter expression was not as uniformly expressed as the Osterix reporter and appeared at higher levels within the trabecular bone region and at lower levels in cortical bone. This is better appreciated at higher magnification (regions of interest are denoted by dashed boxes (b-d) and (e-g)). (b and e) Osterix reporter expression was strongly expressed in osteoblasts and osteocytes within trabecular bone (b) and cortical bone (e). Low Osterix reporter expression was also detected in a cell population present in the bone marrow, but in proximity to trabecular and cortical bone surfaces. (c and f) Bone Sialoprotein reporter expression was strongly detected in osteoblasts and osteocytes in the trabecular bone region (c), but was expressed at lower levels and appeared with less frequency in osteoblasts and osteocytes present in cortical bone (f). Unlike the Osterix reporter, the Bone Sialoprotein reporter was not detected in any cells within the bone marrow. (d and $\mathbf{g}$ ) Merged images reveal the expression of both reporters relative to each other in the trabecular and cortical bone regions. 


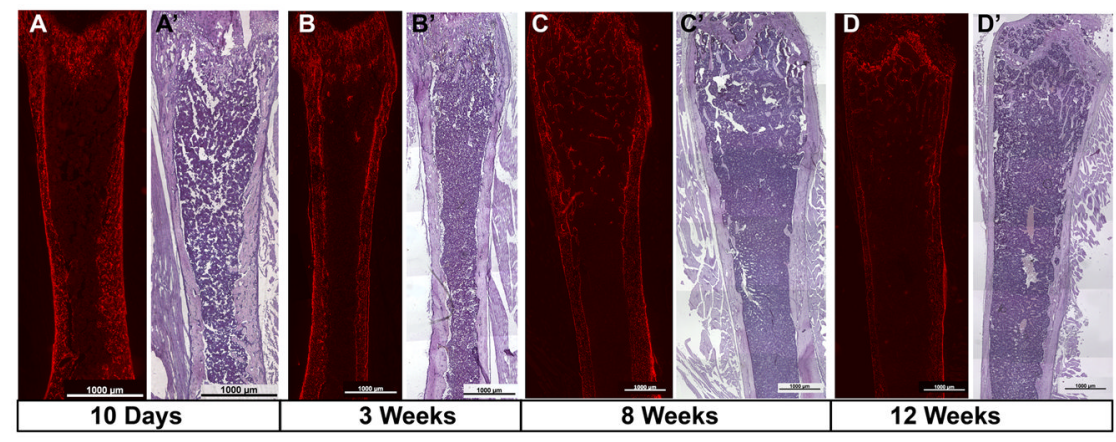

FIG. 4. Strong and Persistent Osterix Reporter Expression in Bone Cells with Aging Osterix reporter expression and corresponding hematoxylin stained tissue sections at

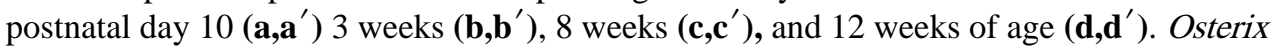
reporter expression persisted with aging in osteoblasts and osteocytes present in the trabecular and cortical bone regions. Fixed exposure times were used and all femurs were taken from male mice. 

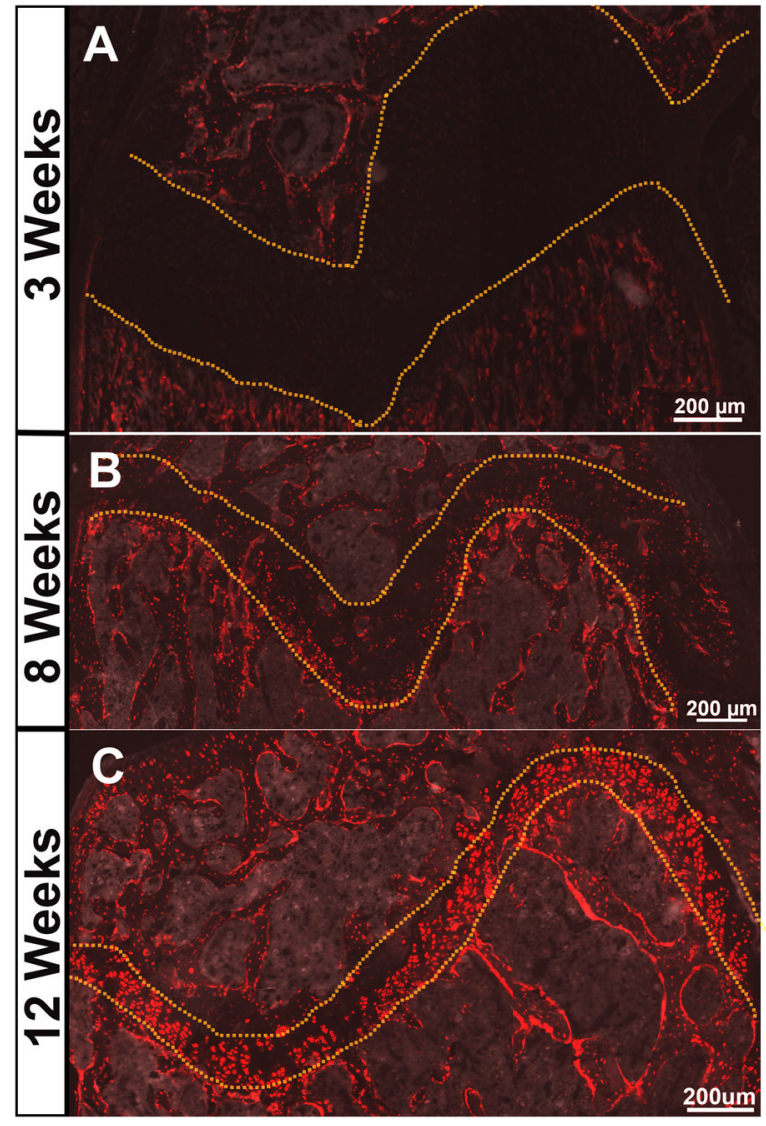

FIG. 5. Increase in Osterix Reporter Expression in Growth Plate Chondrocytes with Aging (a-c) A fixed exposure time was used to compare the fluorescence intensity of Osterix reporter expression in growth plate chondrocytes at 3 weeks (a), 8 weeks (b), and 12 weeks of age (c). While the Osterix reporter can be detected in growth plate chondrocytes in 3 week old mice (a), there is a substantial increase in reporter expression detected in 8 week old mice (b), which continues to increase with age as shown in 12 week old mice (c). 

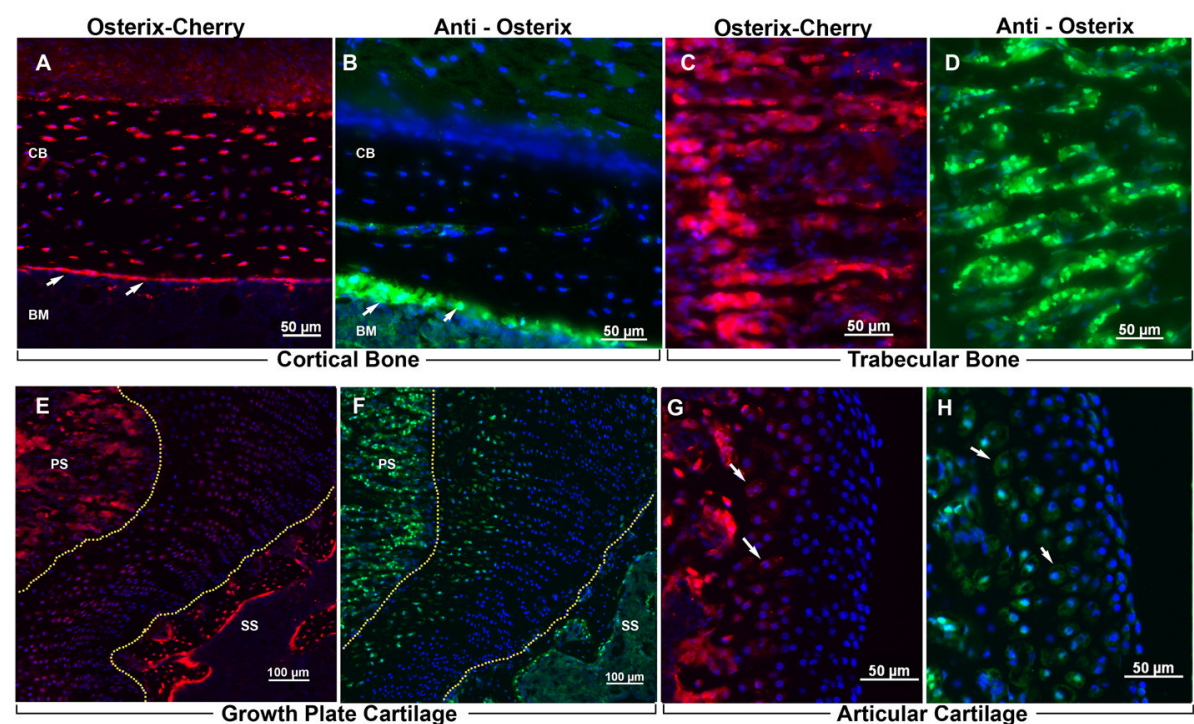

FIG. 6. Comparison of Osterix Reporter Expression to Immunostaining for Osterix Protein Osterix reporter expression (red) was compared to immunostaining for Osterix protein (green) in tissue sections of 4 week old femurs (tissue sections are counterstained with DAPI (blue)). Comparison of expression was assessed in four different regions: (a,b) cortical bone, $(\mathbf{c}, \mathbf{d})$ trabecular bone, $(\mathbf{e , f})$ growth plate cartilage, and $(\mathbf{g , h})$ articular cartilage. Osterix reporter expression was highly expressed in osteoblasts lining the cortical (a, white arrows) and trabecular bone surfaces (c) and osteocytes embedded in bone tissue (a).

Immunostaining also revealed high levels of Osterix protein expression in osteoblasts lining cortical (b, white arrows) and trabecular bone surfaces (d), but was difficult to detect in osteocytes (b). (e) Osterix reporter expression was broadly expressed at low levels in growth plate chondrocytes with slightly higher levels of reporter expression present in maturing chondrocytes. (f) Immunostaining reveals Osterix protein was largely restricted to chondrocytes undergoing hypertrophy. At the end of the distal femur, Osterix reporter expression (g) and immunostaining for Osterix (h) correlate well in the articular cartilage as shown by overlapping signals in chondrocytes undergoing hypertrophy. (BM-bone marrow, CB-cortical bone, PS-primary spongiosa, SS- secondary spongiosa) 

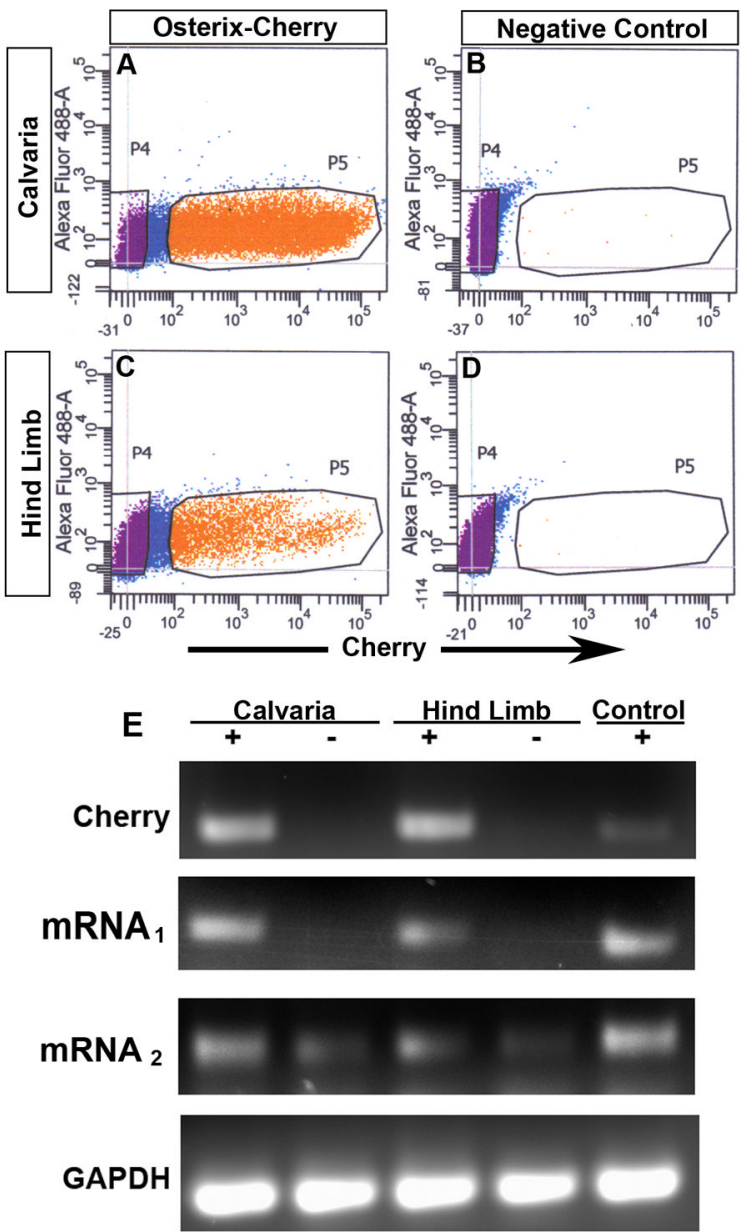

FIG. 7. Gene Expression Analysis of Osterix mRNA Isoforms on FACS Isolated Primary Cells Primary cells were harvested from the calvaria and hind limb of Osterix reporter mice at postnatal day 5 and FACS isolated for gene expression studies. (a-d) FACS isolation of Osterix positive (P5- orange) and negative ( $\mathrm{P} 4$-purple) cell populations from calvaria (a) and hind limb (c). (b,d) Non-transgenic littermate controls are also shown. (e) Gene expression analysis was carried out for the Cherry reporter gene, both transcriptional isoforms of Osterix ( $\mathrm{mRNA}_{1}$ and $\mathrm{mRNA}_{2}$ ) and GAPDH. Both transcriptional isoforms are detected in the Cherry positive cell fraction, while $\mathrm{mRNA}_{2}$ was also weakly detected in the negative cell fraction. 

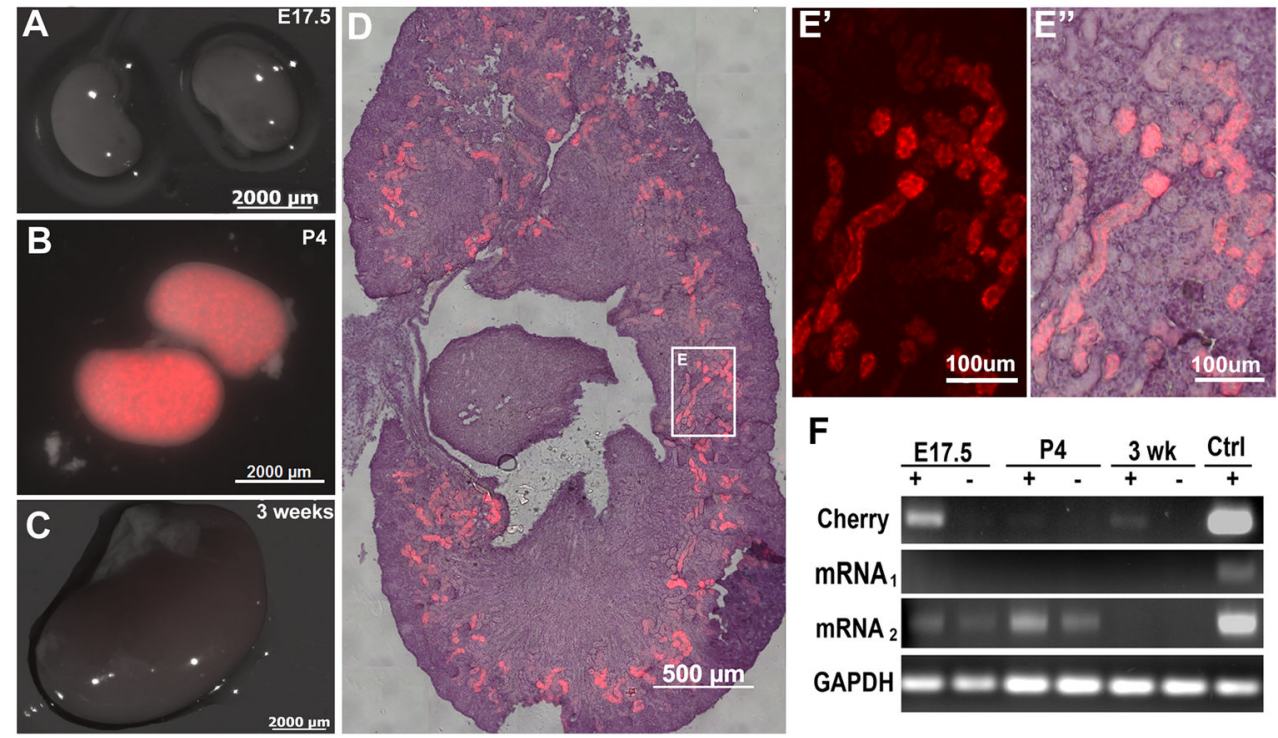

$\mathbf{F}$

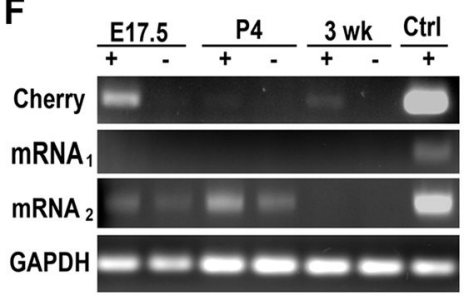

FIG. 8. Transient Osterix Reporter Expression in the Kidney (a-c) Detection of Osterix reporter expression (red) in kidneys harvested from E17.5 (a), post-natal day 4 ( $\mathrm{P} 4)(\mathbf{b})$, and 3 week old (c) animals reveals the transient nature of reporter gene expression. (d, $\left.\mathbf{e}, \mathbf{e}^{\prime}, \mathbf{e}^{\prime \prime}\right)$ Imaging of Osterix reporter expression in kidney tissue sections at P4. (d) Scanned image of a coronal section revealing Osterix reporter expression (red) in the juxtamedullary nephrons (purple-hematoxylin counterstaining). ( $\left.\mathbf{e}^{\prime}, \mathbf{e}^{\prime \prime}\right)$ Reporter expression seen at high magnification from a region of interest shown in (d) revealing expression in the proximal convoluted tubule and the thick descending limb of loop of Henle. (f) Analyses of endogenous Osterix transcription showed that only $\mathrm{mRNA}_{2}$ was transiently detected in the kidney, while expression of mRNA $_{1}$ was not detected. 